\title{
氮硅添加对青藏高原高寒草甸土壤氮矿化的影响
}

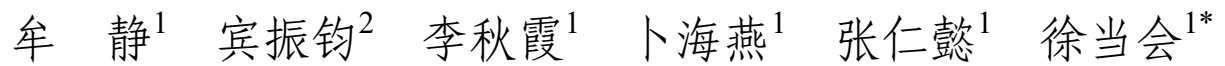

兰州大学生命科学学院, 草地农业生态系统国家重点实验室, 兰州 $730000 ;{ }^{2}$ 广西壮族自治区亚热带作物研究所, 南宁 530001

摘 要 为了解全球气候变化背景下氮沉降对土壤氮矿化的影响及硅添加对土壤氮矿化的促进作用, 该试验设置不同浓度 的氮肥单独添加 $\left(0 、 20 、 40 、 60 \mathrm{~g} \cdot \mathrm{m}^{-2}\right.$, 分别为对照 $\left.\mathrm{CK} 、 \mathrm{~N} 20 、 \mathrm{~N} 40 、 \mathrm{~N} 60\right)$ 以及与硅肥配施(硅酸 $\left.4 \mathrm{~g} \cdot \mathrm{m}^{-2}, \mathrm{Si} 4\right)$, 测定不同处理 下 0-20、20-40、40-60 cm 土层土壤硝态氮含量、铵态氮含量、净硝化速率、净氨化速率以及净矿化速率。结果显示: (1)单 独添加氮肥, 各土层土壤硝态氮和铵态氮含量均随处理浓度的增加而增加, 0-20 cm土层N20、N40、N60处理下土壤硝态氮和 铵态氮分别较CK增加 $63.48 \% 、 126.04 \% 、 247.03 \%$ 和 $80.66 \% 、 152.52 \% 、 244.56 \%$; 随着土层深度增加, 土壤硝态氮、铵态氮 含量均有下降, 20-40、40-60 cm土层较0-20 cm 土层硝态氮含量分别平均减少 $53.90 \% 、 76.05 \%$, 铵态氮含量分别平均减少 $48.62 \% 、 68.23 \%$ 。(2)土壤净硝化速率、净氨化速率及净矿化速率随着氮肥浓度增加均呈上升趋势。相同氮肥添加浓度下, 土 壤净硝化速率、净氨化速率和净矿化速率随着土层深度增加逐渐下降(除CK外)。(3)与单独添加氮肥比较, 氮硅肥配施, 土壤 氮含量有显著提高, 在0-20 cm土层硝态氮和铵态氮较CK分别增加 $98.78 \% 、 192.62 \% 、 330.16 \%$ 和 $99.96 \% 、 195.82 \% 、 306.32 \%$, 20-40、40-60 cm 土层也有类似趋势。同时, 氮硅配施促进了土壤氮矿化行为, 在0-20 cm土层, N60Si4处理下的土壤净硝化速 率、净氨化速率较单独施氮时分别增加 $35.88 \% 、 27.41 \%$ 。以上结果表明, 与单独氮肥添加相比, 氮硅配施不但能提高土壤氮 含量, 而且能促进土壤氮的矿化作用, 对大气氮沉降有一定的缓解作用。

关键词 青藏高原; 养分添加; 硝态氮; 铵态氮; 土壤氮矿化; 氮沉降

牟静, 宾振钧, 李秋霞, 卜海燕, 张仁懿, 徐当会 (2019). 氮硅添加对青藏高原高寒草甸土壤氮矿化的影响. 植物生态学报, 43, 77-84. DOI: $10.17521 /$ cjpe.2018.0218

\section{Effects of nitrogen and silicon addition on soil nitrogen mineralization in alpine meadows of Qinghai-Xizang Plateau}

MOU Jing ${ }^{1}$, BIN Zhen-Jun ${ }^{2}$, LI Qiu-Xia ${ }^{1}$, BU Hai-Yan ${ }^{1}$, ZHANG Ren-Yi ${ }^{1}$, and XU Dang-Hui ${ }^{1 *}$

${ }^{1}$ School of Life Science, Lanzhou University, State Key Laboratory of Grassland Agro-ecosystems, Lanzhou 730000, China; and ${ }^{2}$ Guangxi Subtropical Crops Research Institute, Nanning 530001, China

\section{Abstract}

Aims To understand the effects of nitrogen and silicon addition on soil nitrogen mineralization in the context of global climate change.

Methods Nitrogen fertilizer with different concentration were added separately $\left(0,20,40,60 \mathrm{~g} \cdot \mathrm{m}^{-2}\right.$, namely CK, $\mathrm{N} 20, \mathrm{~N} 40, \mathrm{~N} 60$, respectively) and combined with silicon fertilizer $\left(4 \mathrm{~g} \cdot \mathrm{m}^{-2}\right.$ silicic acid, namely $\left.\mathrm{Si4}\right)$. Soil nitrate and ammonium nitrogen contents, net nitrification rate, net ammonification rate as well as net mineralization rate in 0-20, 20-40 and 40-60 cm depth profiles were measured.

Important findings (1) When nitrogen fertilizer was applied alone, soil nitrate and ammonium nitrogen contents under N20, N40, N60 treatments in 0-20 cm soil layer increased by $63.48 \%, 126.04 \%, 247.03 \%$ and $80.66 \%$, $152.52 \%, 244.56 \%$ respectively, compared with CK. With the increase of soil depth, soil nitrate and ammonium nitrogen both decreased, the nitrate nitrogen content in 20-40 and 40-60 cm soil layers decreased by an average of $53.90 \%, 76.05 \%$ respectively and the ammonium nitrogen content decreased by an average of $48.62 \%, 68.23 \%$ respectively, compared with $0-20 \mathrm{~cm}$ soil layer. (2) With the increase of $\mathrm{N}$ application, soil net nitrification rate, net ammoniation rate and net mineralization rate all showed an increasing trend. Under the same nitrogen fertilizer concentration, with the increase of soil depth, net nitrification rate, net ammonification rate and net mineralization rate gradually decreased (except for CK). (3) Compared with nitrogen addition alone, soil nitrogen content was

收稿日期Received: 2018-09-11 接受日期Accepted: 2018-10-31

基金项目: 国家重点研发计划(2016YFC0501906)和国家自然科学基金(30900171、31460162和31600336)。Supported by the National Key R\&D Program (2016 YFC0501906), and the National Natural Science Foundation (30900171, 31460162 and 31600336).

* 通信作者Corresponding author (dhxu@lzu.edu.cn) 
significantly improved when nitrogen and silicon fertilizers were both applied, in the 0-20 $\mathrm{cm}$ soil layer, the increase ratio of nitrate nitrogen and ammonium nitrogen were $98.78 \%, 192.62 \%, 330.16 \%$ and $99.96 \%$, $195.82 \%, 306.32 \%$ respectively, compared with CK. This trend was also found in the $20-40$ and $40-60 \mathrm{~cm}$ soil layers. The results also showed that combined application of nitrogen and silicon had a significant effect on soil nitrogen mineralization. For example, net nitrification rate and net ammonification rate under N60Si4 treatment increased by $35.88 \%$ and $27.41 \%$ respectively in the $0-20 \mathrm{~cm}$ soil layer, compared with nitrogen application alone. The above results showed that compared with the nitrogen addition alone, the application of both nitrogen and silicon can not only increase the nitrogen content in different soil layers, but also promote the mineralization of soil nitrogen and therefore having a certain mitigation effect on atmospheric nitrogen deposition.

Key words Qinghai-Xizang Plateau; nutrients addition; nitrate nitrogen; ammonium nitrogen; soil nitrogen mineralization; nitrogen deposition

Mou J, Bin ZJ, Li QX, Bu HY, Zhang RY, Xu DH (2019). Effects of nitrogen and silicon addition on soil nitrogen mineralization in alpine meadows of Qinghai-Xizang Plateau. Chinese Journal of Plant Ecology, 43, 77-84. DOI: 10.17521/cjpe.2018.0218

青藏高原平均海拔在4 $000 \mathrm{~m}$ 以上, 有“世界屋 脊”之称, 分布有世界海拔最高的草地。作为青藏高 原自然生态系统的主体(刘兴元等, 2012), 高寒草地 面积达 $1.525 \times 10^{8} \mathrm{hm}^{2}$, 占青藏高原总面积的 $59.28 \%$ (张镱锂等, 2013)。由于海拔高、气候寒冷、干旱等 因素, 该地区有机氮的利用率低, 仅占土壤全氮的 1\% (白洁冰等, 2011)。近年来, 随着青藏高原东缘区 域性氮沉降逐年增加, 该地区土壤中的可用氮增加 (杨晓霞等, 2014), 继而对土壤中氮的活动产生影 响。添加营养元素是维持草地生态系统养分平衡, 减缓草地退化的重要措施, 添加氮能有效提高土壤 有效氮含量, 促进草地生态系统养分循环(刘晓东 等, 2015)。硅对植物生长同样至关重要, 不仅可增 加植物抗逆、抗倒伏能力, 还可活化土壤有机质, 有 效促进植物对氮、磷等的吸收(张文鹏等, 2016)。但 之前的研究多集中于硅对植物群落多样性、植物抗 性等方面的影响, 而对土壤氮矿化的影响研究较 少。本研究将土壤矿化作为重点, 探讨氮硅添加对 土壤矿化的影响, 以期为维持青藏高原草地生态系 统的平衡和制定适当管理措施提供有效的理论依 据。

\section{1 研究区域和方法}

研究样地设于兰州大学高寒草甸与湿地生态系 统定位研究站(阿孜分站), 位于青藏高原东缘甘南 藏族自治州玛曲县 $\left(33.65^{\circ} \mathrm{N}, 101.88^{\circ} \mathrm{E}\right)$ 。海拔 $3650 \mathrm{~m}$, 年平均气温 $2.2{ }^{\circ} \mathrm{C}$, 年降水量 $672 \mathrm{~mm}$, 降 水集中在7-8月, 属于高寒半湿润半干旱气候。年日 照时间约 $2580 \mathrm{~h}$, 年霜期大于 270 天。样地内的优势 种主要有嵩草(Kobresia myosuroides)、垂穗披碱草
(Elymus nutans)、草地早熟禾(Poa pratensis)、长毛 风毛菊(Saussurea hieracioides)、星状雪兔子 ( $S$. stella)、针苍雪莲(S. nigrescens)、高原毛莨(Ranunculus tanguticus)、针裂银莲花(Anemone obtusiloba)、草玉梅(A. rivularis)、中国马先蒿(Pedicularis chinensis)、短腺小米草(Euphrasia regelii)、莓叶委 陵菜(Potentilla fragarioides)、多枝黄耆(Astragalus polycladus)、黄花棘豆(Oxytropis ochrocephala)等。

\section{1 样地设计与处理}

样地选自禁牧区, 四周采用围栏围起来以防止 牛羊进入破坏。试验采取完全区组设计。在样地内 选取 48 个 $2 \mathrm{~m} \times 2 \mathrm{~m}$ 的样方, 各样方之间留 $2 \mathrm{~m}$ 间隔, 每个样方四角用PVC管标记。处理前进行土壤采样, 在每个样方内随机选取 3 个采样点, 使用 $5 \mathrm{~cm}$ 孔径 的土钻分别钻取 0-20、20-40、40-60 cm深度的土样, 装入自封袋带回实验室, 分析其理化性质, 作为土 壤氮矿化的初始值。于2012年5月中旬进行氮、硅添 加试验, 均为一次性均匀施肥。根据以往在该地的 长期施肥试验资料(刘慧霞等, 2009; 张杰琦等, 2010)并结合实际情况, 本试验设置4组处理, 分别 为对照 $\left(\mathrm{CK}\right.$, 不添加 $\left.\mathrm{NH}_{4} \mathrm{NO}_{3} 、 \mathrm{H}_{2} \mathrm{SiO}_{3}\right)$, 单独氮添加 处理(添加 $\mathrm{NH}_{4} \mathrm{NO}_{3} 20 、 40 、 60 \mathrm{~g} \cdot \mathrm{m}^{-2}$, 分别记为 $\mathrm{N} 20$ 、 $\mathrm{N} 40 、 \mathrm{~N} 60$, 折合施氮量分别为 $7 、 14 、 21 \mathrm{~g} \cdot \mathrm{m}^{-2}$ ), 单 独硅添加处理(添加 $\mathrm{H}_{2} \mathrm{SiO}_{3} 4 \mathrm{~g} \cdot \mathrm{m}^{-2}$, 即 $\mathrm{Si} 4$, 折合施硅 量 $1.436 \mathrm{~g} \cdot \mathrm{m}^{-2}$ ), 氮、硅配施处理(在N20、N40、N60 基础上, 添加硅酸 $4 \mathrm{~g} \cdot \mathrm{m}^{-2}$, 分别记为 $\mathrm{N} 20 \mathrm{Si}$, $\mathrm{N} 40 \mathrm{Si} 4, \mathrm{~N} 60 \mathrm{Si} 4$ ), 共计 8 个处理, 每处理6个重复。处 理前土壤 $\mathrm{pH}$ 值 6.33 , 有机质含量 $70.52 \mathrm{~g} \cdot \mathrm{kg}^{-1}$, 全氮 含量 $3.72 \mathrm{~g} \cdot \mathrm{kg}^{-1}$, 铵态氮、硝态氮含量分别为 10.67 $\mathrm{mg} \cdot \mathrm{kg}^{-1}$ 和 $15.25 \mathrm{mg} \cdot \mathrm{kg}^{-1}$; 全磷含量 $0.98 \mathrm{~g} \cdot \mathrm{kg}^{-1}$, 速 
效磷含量 $25.27 \mathrm{mg} \cdot \mathrm{kg}^{-1}$ 。

于 2012 年 8 月中旬, 在每个样方内随机选取 3 个 采样点, 按0-20、20-40、40-60 cm 土层深度, 依次 用 $5 \mathrm{~cm}$ 孔径的土钻取样, 装入自封袋, 带回实验室 进行分析，作为土壤氮矿化的处理值。

\section{2 测定方法与数据处理}

按常规分析方法测定土壤理化性质。利用 $\mathrm{pH}$ 仪 (PHS-5, 上海艾测电子科技有限公司，上海)测定土 壤 $\mathrm{pH}$ 值; 采用重铬酸钾容量法测定土壤有机质含量; 土样分别采用 $\mathrm{H}_{2} \mathrm{SO}_{4}-\mathrm{K}_{2} \mathrm{SO}_{4}: \mathrm{CuSO}_{4}: \mathrm{Se}$ 法和 $\mathrm{HClO}_{4}{ }^{-}$ $\mathrm{H}_{2} \mathrm{SO}$ 法催化消煮, 消煮后的溶液经定容、沉淀和稀 释后采用SmartChem 200 全自动智能化学分析仪 (WestCo Scientific Instruments, Brookfield, USA)进 行全氮、全磷含量分析测定; 采用钼锑抗比色法测 定土壤速效磷含量。土壤硝态氮和铵态氮用 $2 \mathrm{~mol} \cdot \mathrm{L}^{-1} \cdot \mathrm{KCl}$ 溶液浸提后, 用 $\mathrm{SmartChem} 200$ 全自 动智能化学分析仪测定其含量。依照下式计算土壤 氮素矿化速率:

净硝化速率 $=\left(\right.$ 处理后 $\mathrm{NO}_{3}^{-}-\mathrm{N}-$ 初始 $\left.\mathrm{NO}_{3}^{-}-\mathrm{N}\right) /$ 培养天数

净氨化速率 $=\left(\right.$ 处理后 $\mathrm{NH}_{4}^{+}-\mathrm{N}-$ 初始 $\left.\mathrm{NH}_{4}^{+}-\mathrm{N}\right) /$ 培养天数

净矿化速率 $=\left(\left(\right.\right.$ 处理后 $\mathrm{NH}_{4}^{+}-\mathrm{N}+$ 处理后 $\left.\mathrm{NO}_{3}^{-}-\mathrm{N}\right)-\left(\right.$ 初始 $\mathrm{NH}_{4}^{+}-\mathrm{N}+$ 初始 $\left.\left.\mathrm{NO}_{3}^{-}-\mathrm{N}\right)\right)$ /培养天数

采用Excel 2010、SPSS 22.0等软件对数据进行 计算和处理, 采用Origin 8.5 作图。不同处理间差异 采用单因素方差分析检验; 对土壤深度和施肥处理
对土壤净矿化速率的影响进行双因素方差分析，显 著性差异水平为 $p<0.05$ 。

\section{2 结果}

\section{1 氮硅添加对土壤硝态氮、铵态氮含量的影响}

在N20、N40、N60处理下, 0-20 cm 土层硝态氮 含量较 $\mathrm{CK}$ 分别增加 $63.48 \%$ 、 $126.04 \%$ 和 $247.03 \%$; 20-40 cm 土层较CK分别增加 $55.57 \% 、 138.20 \%$ 和 $247.86 \%$; 40-60 cm 土层较CK分别增加 $52.93 \%$ 、 $83.55 \%$ 和 $89.55 \%$ 。随着土壤深度增加, 各处理下土 壤硝态氮含量均呈现下降趋势, 20-40、40-60 cm 土 层较 $0-20 \mathrm{~cm}$ 土层硝态氮含量分别平均减少 $53.90 \%$ 和 $76.05 \%$ (图1A)。氮硅配施时, 随着氮肥浓度的增 加土壤硝态氮含量显著增加 $(p<0.05), 0-20 \mathrm{~cm}$ 土层 较CK分别增加 $98.78 \%$ 、192.62\%和 $330.16 \% ; 20-40$ $\mathrm{cm}$ 土层分别增加 $88.06 \%$ 、167.62\%和 $299.70 \% ; 40-60$ $\mathrm{cm}$ 土层分别增加 $51.43 \%$ 、 $89.18 \%$ 和 $136.92 \%$ (图 1B)。养分处理对土壤硝态氮含量的影响随着土壤深 度的增加逐渐减弱。

单独添加氮肥时，0-20 cm 土层铵态氮含量较 CK分别增加 $80.66 \% 、 152.52 \%$ 和 $244.56 \%$ 。20-40、 40-60 $\mathrm{cm}$ 铵态氮含量较 $\mathrm{CK}$ 分别增加 $59.94 \%$ 、 $56.22 \% 、 132.30 \%$ 和 $129.81 \% 、 217.79 \% 、 176.37 \%$ 。 随着土壤深度的增加, 土壤铵态氮含量逐渐减少, 20-40、40-60 cm 土层较0-20 cm 分别平均减少 $48.62 \% 、 68.23 \%$ (图2A)。当氮硅配施时, 各土层铵 态氮含量较硝态氮含量增幅更大, 0-20 cm 土层增加
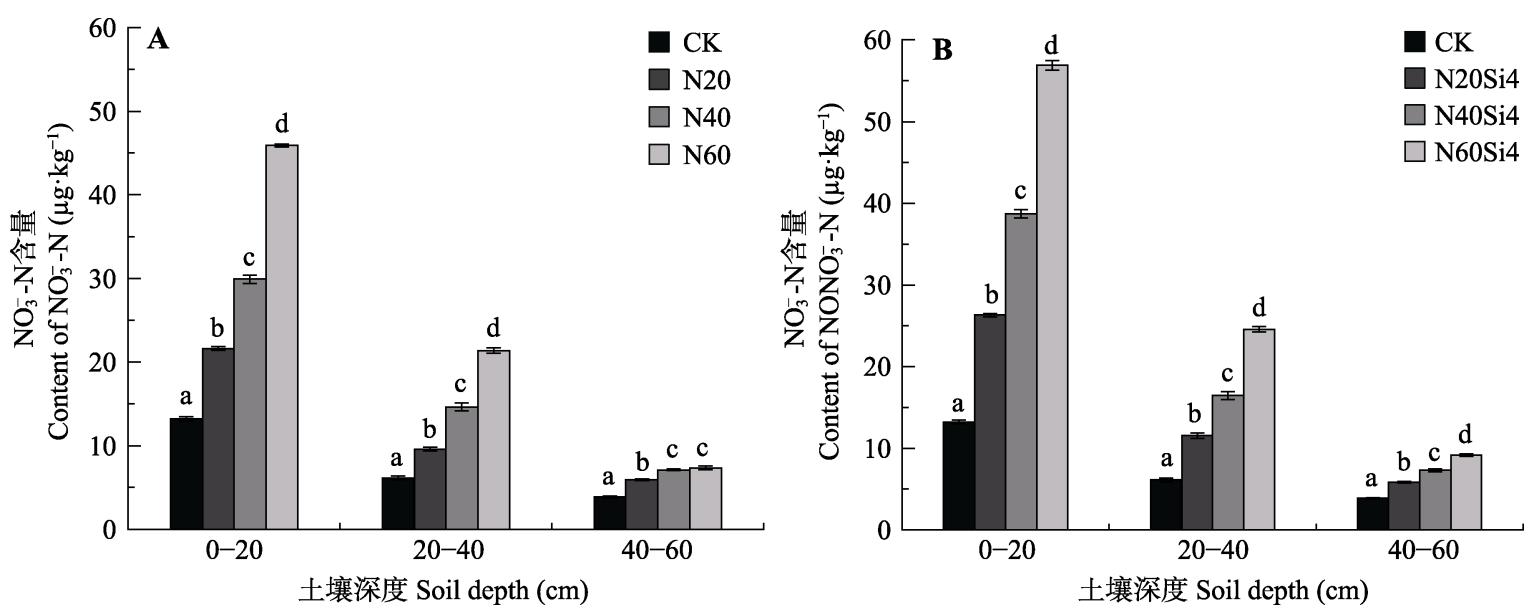

图1 青藏高原高寒草甸不同施氮浓度下不同土层硝态氮含量变化(平均值沶准误差, $n=6$ )。CK、N20、N40、N60, 分别添 加 $\mathrm{NH}_{4} \mathrm{NO}_{3}$ 0、20、40、60 g $\cdot \mathrm{m}^{-2} ; \mathrm{Si} 4$, 添加硅酸 $4 \mathrm{~g} \cdot \mathrm{m}^{-2}$ 。不同小写字母表示处理间差异显著 $(p<0.05)$ 。

Fig. 1 Change of $\mathrm{NO}_{3}^{-}-\mathrm{N}$ content in alpine meadows of Qinghai-Xizang Plateau under different nitrogen concentrations (mean $\pm \mathrm{SE}$, $n=6)$. CK, N20, N40, N60, added $\mathrm{NH}_{4} \mathrm{NO}_{3} 0,20,40,60 \mathrm{~g} \cdot \mathrm{m}^{-2}$, respectively; Si4, added silicic acid $4 \mathrm{~g} \cdot \mathrm{m}^{-2}$. Different lowercase letters indicate significant differences among treatments $(p<0.05)$. 
$99.96 \% 、 195.82 \%$ 和 $306.32 \% ; 20-40 、 40-60 \mathrm{~cm}$ 土层 铵态氮含量分别增加 $82.22 \% 、 175.06 \% 、 291.49 \%$ 和 $82.45 \% 、 164.09 \% 、 225.67 \%$ (图2B)。随着土壤深 度增加, 各养分处理对土壤铵态氮含量的影响也逐 渐减弱。

\section{2 氮硅添加对土壤氮矿化的影响}

如图3A所示, 施加氮肥前, 各土层净硝化速率 均为负值, 其中 $20-40 \mathrm{~cm}$ 土壤净硝化速率达最小值 $-26.65 \mu \mathrm{g} \cdot \mathrm{kg}^{-1} \cdot \mathrm{d}^{-1}$ 。单独施加氮肥时, 各土层净硝化 速率随着施氮水平的增加呈上升趋势, 其中 0-20 cm土层增幅最大, 20-40 $\mathrm{cm}$ 次之, 40-60 $\mathrm{cm}$ 增 幅最小(图3 A)。在施硅肥的基础上，逐浓度增施氮
肥，各土层净硝化速率显著增加，其最大值462.61 $\mu \mathrm{g} \cdot \mathrm{kg}^{-1} \cdot \mathrm{d}^{-1}$ 出现在N60Si4处理下 $0-20 \mathrm{~cm}$ 土层, 且各 土层增幅与单独施加氮肥时类似, 0-20 cm 土层增幅 最大, 20-40 cm次之, 40-60 cm 增幅最小(图3B)。 0-20、20-40 cm 土层净硝化速率均高于单独添加氮 肥时的净硝化速率, 但在 $40-60 \mathrm{~cm}$ 土层, N20Si4处 理时则出现了后者高于前者的情况。

如图4A所示, 氮处理前, 各土层净氨化速率均 为负值, 其中在 $0-20 \mathrm{~cm}$ 达最小值 $-22.63 \mu \mathrm{g} \cdot \mathrm{kg}^{-1} \cdot \mathrm{d}^{-1}$, 且随土壤深度的增加, 净氨化速率逐渐增大。单独 添加氮肥时, 各土层的净氨化速率随氮肥浓度的增 加而增加, 其中0-20 cm 土层增幅较20-40、40-60 cm
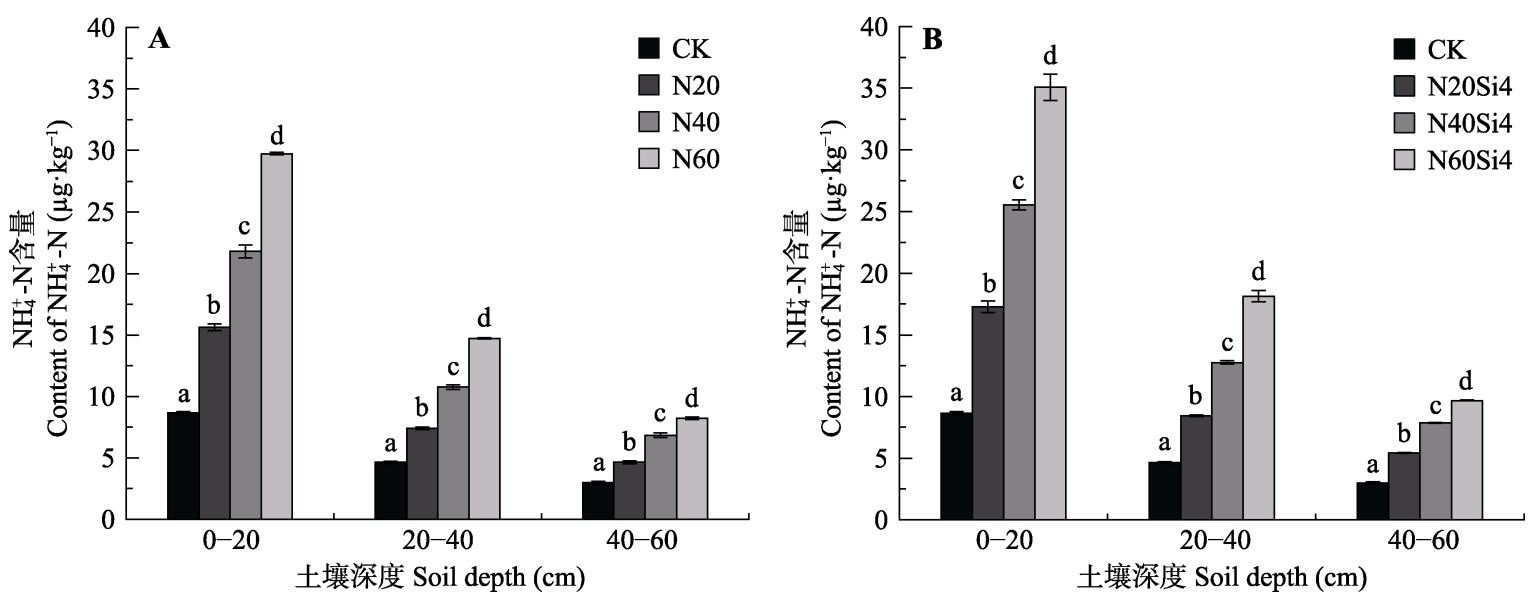

图2 青藏高原高寒草甸不同施氮浓度下不同土层铵态氮含量变化(平均值沶准误差, $n=6$ )。CK、N20、N40、N60, 分别添 加 $\mathrm{NH}_{4} \mathrm{NO}_{3}$ 0、20、40、60 g $\cdot \mathrm{m}^{-2} ; \mathrm{Si} 4$, 添加硅酸 $4 \mathrm{~g} \cdot \mathrm{m}^{-2}$ 。不同小写字母表示处理间差异显著 $(p<0.05)$ 。

Fig. 2 Change of $\mathrm{NH}_{4}^{+}-\mathrm{N}$ content in alpine meadows of Qinghai-Xizang Plateau under different nitrogen concentrations (mean $\pm S E$, $n=6)$. CK, N20, N40, N60, added $\mathrm{NH}_{4} \mathrm{NO}_{3} 0,20,40,60 \mathrm{~g} \cdot \mathrm{m}^{-2}$, respectively; Si4, added silicic acid $4 \mathrm{~g} \cdot \mathrm{m}^{-2}$. Different lowercase letters indicate significant differences among treatments $(p<0.05)$.
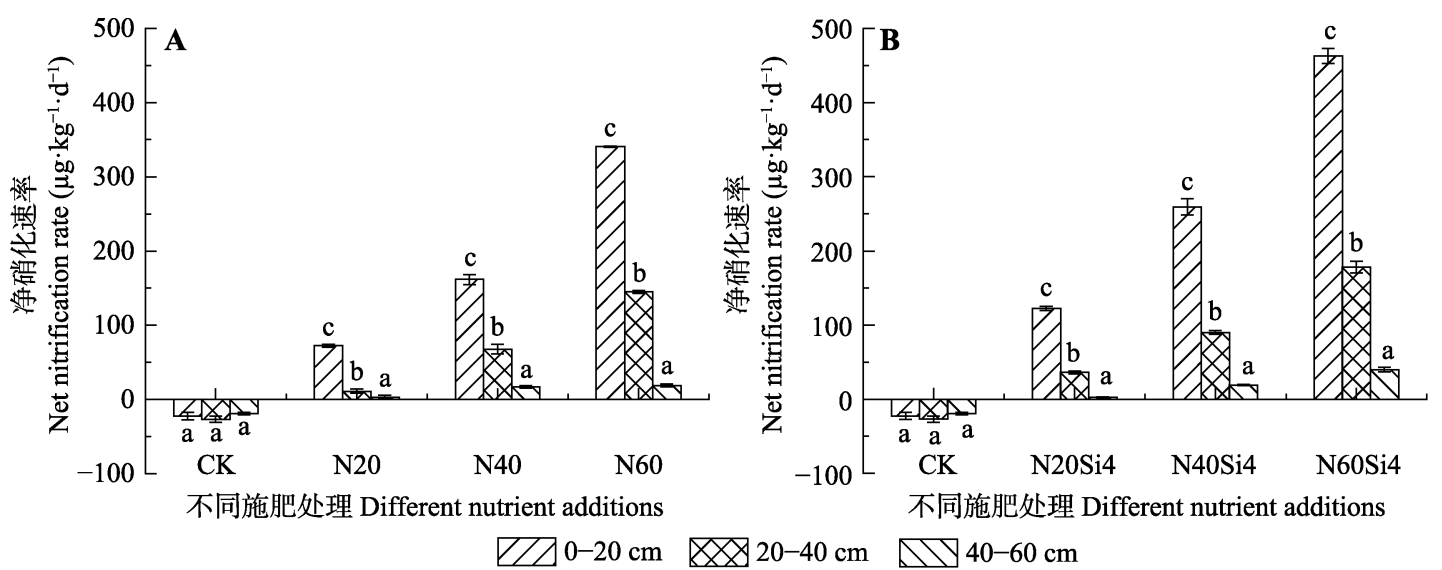

图3 青藏高原高寒草甸不同施氮浓度下土壤净硝化速率(平均值 \pm 标准误差, $n=6$ )。CK、N20、 $440 、 \mathrm{~N}^{\prime} 6$, 分别添加 $\mathrm{NH}_{4} \mathrm{NO}_{3}$ 0、20、40、60 $\mathrm{g} \cdot \mathrm{m}^{-2} ; \mathrm{Si} 4$ ，添加硅酸 $4 \mathrm{~g} \cdot \mathrm{m}^{-2}$ 。不同小写字母表示处理间差异显著 $(p<0.05)$ 。

Fig. 3 Net nitrification rates under different nitrogen concentrations in alpine meadows of Qinghai-Xizang Plateau (mean $\pm S E, n=$ 6). CK, N20, N40, N60, added $\mathrm{NH}_{4} \mathrm{NO}_{3} 0,20,40,60 \mathrm{~g} \cdot \mathrm{m}^{-2}$, respectively; Si4, added silicic acid $4 \mathrm{~g} \cdot \mathrm{m}^{-2}$. Different lowercase letters indicate significant differences among treatments $(p<0.05)$. 
土层大。以氮硅配施的方法处理, 土壤净氨化速率 最大值270.66 $\mu \mathrm{g} \cdot \mathrm{kg}^{-1} \cdot \mathrm{d}^{-1}$ 同样出现在0-20 $\mathrm{cm}$ 土层, 且氮硅配施时各土层净氨化速率均大于单氮施加时 的净氨化速率。

土壤净矿化速率在氮处理前均为负值, 且土壤 净矿化速率随土层深度的增加而增加, 但始终小于 0 (图5A)。单独施加氮肥时, 0-20 cm 土层、 $\mathrm{N} 60$ 处理 下的净矿化速率达 $552.89 \mu \mathrm{g} \cdot \mathrm{kg}^{-1} \cdot \mathrm{d}^{-1}$, 较 $\mathrm{N} 20$ 增加 $335.69 \%$ 。氮硅配施, 土壤净矿化速率较单独施加氮 肥时显著增加，在各土层中， N20Si4、N40Si4、 N60Si4分别较N20、N40、N60处理下的土壤矿化速 率增加 $55.16 \% 、 112.86 \% 、 61.19 \%$ (图5B)。

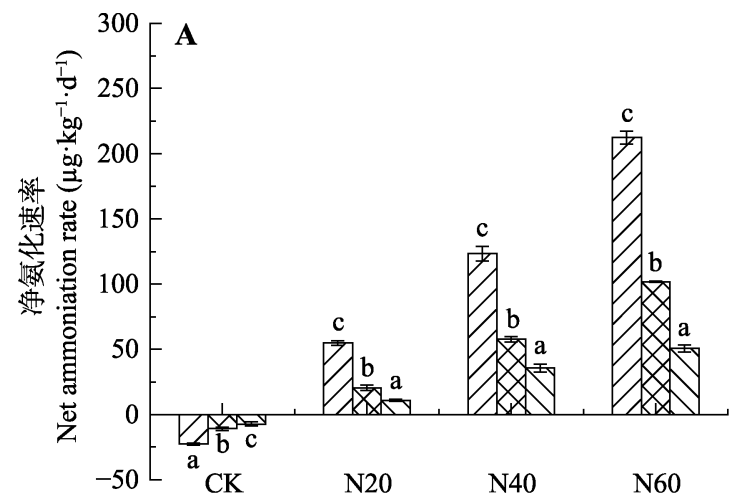

不同施肥处理 Different nutrient additions
不同施肥处理下各土层间土壤净硝化速率(除 $\mathrm{CK}$ 外)、净氨化速率和净矿化速率间均存在显著性 差异 $(p<0.05$ )(图3-5), 故除施肥外, 土壤深度也是 影响土壤矿化行为的关键因子, 且两者对土壤净矿 化速率存在交互作用 $(p<0.05)$ (表1)。

\section{3 讨论}

氮矿化的研究对于揭示生态系统功能, 为维持 生态系统稳定提供理论指导有重要意义。氮是植物 生长发育必需的营养元素, 是植物吸收量最大的土 壤矿质元素(赵阳等, 2013), 也是影响整个陆地系统 生产力的重要因子, 通常被认为是植物生长发育重

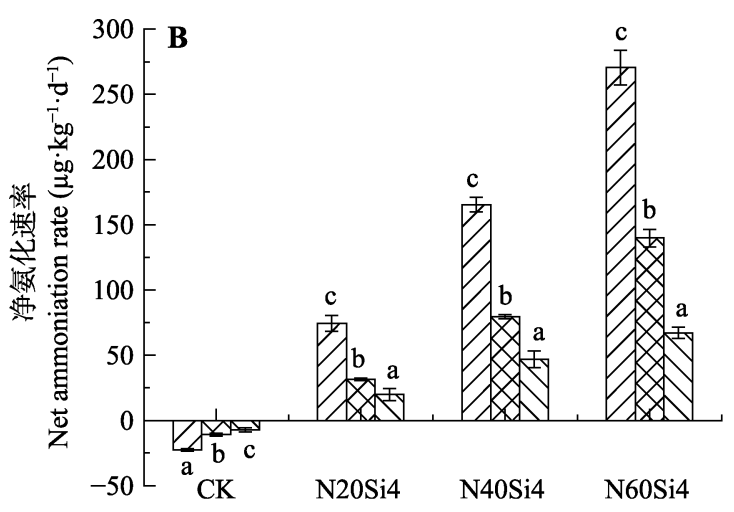

不同施肥处理 Different nutrient additions

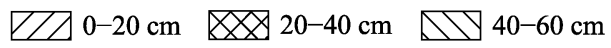

图4 青藏高原高寒草甸不同施氮浓度下土壤净氨化速率(平均值土标准误差, $n=6$ )。CK、N20、 $\mathrm{N} 40 、 \mathrm{~N}^{\circ}$, 分别添加 $\mathrm{NH}_{4} \mathrm{NO}_{3}$ 0、20、40、60 $\mathrm{g} \cdot \mathrm{m}^{-2} ; \mathrm{Si} 4$, 添加硅酸 $4 \mathrm{~g} \cdot \mathrm{m}^{-2}$ 。不同小写字母表示处理间差异显著 $(p<0.05)$ 。

Fig. 4 Net ammonification rates under different nitrogen concentrations in alpine meadows of Qinghai-Xizang Plateau (mean $\pm S E$, $n=6)$. CK, N20, N40, N60, added $\mathrm{NH}_{4} \mathrm{NO}_{3} 0,20,40,60 \mathrm{~g} \cdot \mathrm{m}^{-2}$, respectively; Si4, added silicic acid $4 \mathrm{~g} \cdot \mathrm{m}^{-2}$. Different lowercase letters indicate significant differences among treatments $(p<0.05)$.
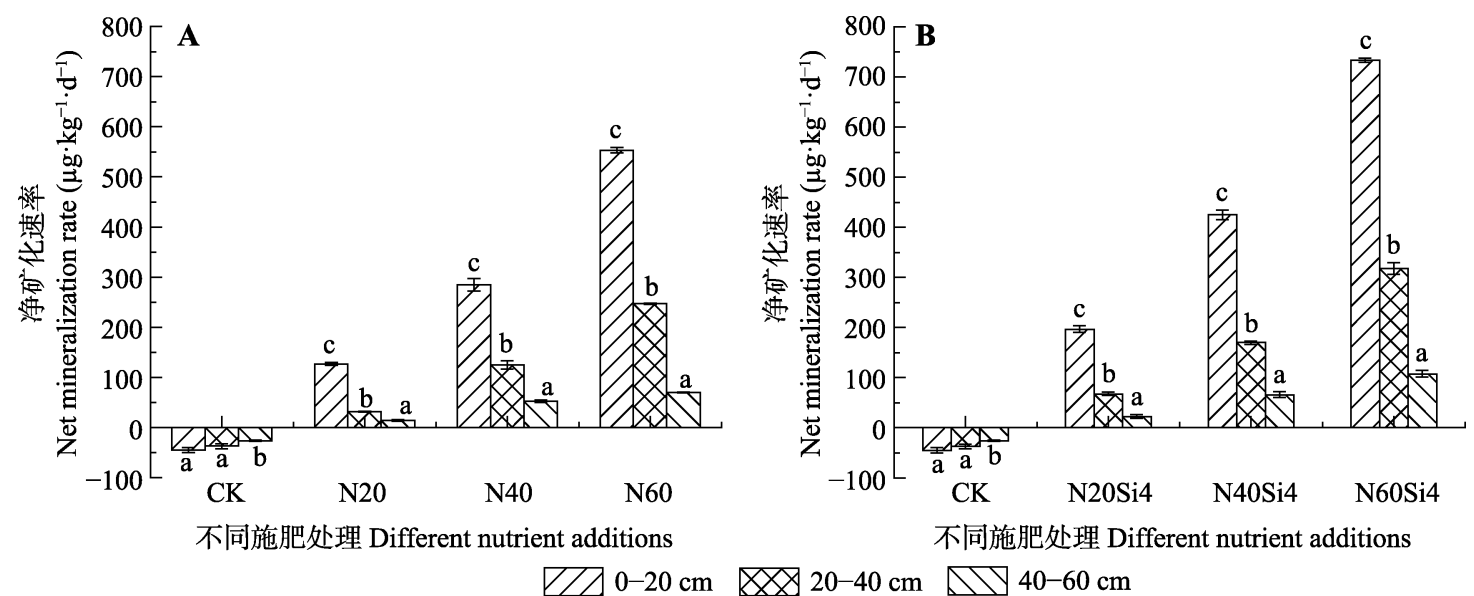

图5 青藏高原高寒草甸不同施氮浓度下土壤净矿化速率(平均值土标准误差, $n=6$ )。CK、N20、 $\mathrm{N} 40 、 \mathrm{~N}^{\circ}$, 分别添加 $\mathrm{NH}_{4} \mathrm{NO}_{3}$ $0 、 20 、 40 、 60 \mathrm{~g} \cdot \mathrm{m}^{-2} ; \mathrm{Si} 4$ ，添加硅酸 $4 \mathrm{~g} \cdot \mathrm{m}^{-2}$ 。不同小写字母表示处理间差异显著 $(p<0.05)$ 。

Fig. 5 Net mineralization rates under different nitrogen concentrations in alpine meadows of Qinghai-Xizang Plateau (mean $\pm S E, n$ $=6$ ). $\mathrm{CK}, \mathrm{N} 20, \mathrm{~N} 40, \mathrm{~N} 60$, added $\mathrm{NH}_{4} \mathrm{NO}_{3} 0,20,40,60 \mathrm{~g} \cdot \mathrm{m}^{-2}$, respectively; Si4, added silicic acid $4 \mathrm{~g} \cdot \mathrm{m}^{-2}$. Different lowercase letters indicate significant differences among treatments $(p<0.05)$. 
表1 施肥、土壤深度对青藏高原高寒草甸土壤净矿化速率的双因素方差分析结果

Table 1 Two-factor variance analysis between fertilization and soil depths on soil net mineralization rate in alpine meadows of Qinghai-Xizang Plateau

\begin{tabular}{|c|c|c|c|c|c|}
\hline 来源 Source & 第三类平方和 Type III sum of squares & $d f$ & 均方 Mean Square & $F$ & 显著性 Sig. \\
\hline 校正模型 Corrected Model & $1436023.182^{\mathrm{a}}$ & 8 & 179502.898 & 5325.359 & 0.000 \\
\hline 截距 Intercept & 1238798.353 & 1 & 1238798.353 & 36751.753 & 0.000 \\
\hline $\mathrm{NSi}$ & 906007.721 & 2 & 453003.860 & 13439.383 & 0.000 \\
\hline SD & 318252.753 & 2 & 159126.376 & 4720.844 & 0.000 \\
\hline $\mathrm{NSi} \times \mathrm{SD}$ & 211762.709 & 4 & 52940.677 & 1570.605 & 0.000 \\
\hline 误差 Error & 606.729 & 18 & 33.707 & & \\
\hline 总计 Total & 2675428.265 & 27 & & & \\
\hline 校正后总数 Corrected total & 1436629.912 & 26 & & & \\
\hline
\end{tabular}

$\mathrm{a}, R^{2}=1.000$ (调整的 $R^{2}=0.999$ )。 $\mathrm{NSi}$, 氮硅配施; $\mathrm{SD}$, 土壤深度。

a, $R^{2}=1.000$ (adjusted $R^{2}=0.999$ ). NSi, nitrogen and silicon combined; $\mathrm{SD}$, soil depth.

要限制因素(司晓林等, 2016)。氮在土壤中绝大部分 是以有机形式存在的, 约占土壤全氮含量的 $92 \%-98 \%$, 土壤有机氮无法直接被植物吸收利用, 必须借助土壤微生物, 通过矿化作用将其转化为无 机氮。土壤中无机氮主要形态有铵态氮和硝态氮, 二者都具水溶性，更易被植物吸收利用。自18世纪 后期, 土壤氮矿化已被视为土壤氮循环的核心, 在 控制植物有效氮利用方面起到至关重要的作用 (Aber \& Melillo, 2001)。

本研究结果表明, 施加氮肥可提高土壤有效氮 含量, 即硝态氮和铵态氮的含量, 且浅层土壤 $(0-20$ $\mathrm{cm}$ )的响应较深层土壤(20-40、40-60 $\mathrm{cm}$ )强烈(图1, 图2), 这与郭雅婧等(2015)的研究结果一致。铵态氮 主要以交换态形式存在, 较小的土粒 $(<0.001 \mathrm{~mm})$ 一般呈负电性, 可以吸附交换态的铵态氮, 因此, 土壤中铵态氮的移动范围一般都较小; 相反, 硝态 氮呈负电性, 难以被土粒吸附, 在土壤中移动范围 较铵态氮大, 故在各土层分布应相对均匀(奚振邦, 2011)。但在本研究中, 硝态氮和铵态氮在各土层的 分布情况类似, 即在氮处理后, 二者含量均随着土 层深度的增加呈现出递减的趋势。可能的解释是硝 态氮在土壤中活动性强, 有利于植物深层的根系吸 收, 但也易流失。

施肥可促进高寒草甸土壤的矿化作用, 且与土 壤深度对土壤的矿化作用存在交互作用(表1), 就本 试验而言, 0-20 cm 土层矿化行为较20-40、40-60 cm 土层强烈, 这与Aber等(1989)、Gundersen等(1998) 对森林生态系统土壤的研究结果一致。但随着研究 的不断深入, Aber和Magill (2004)发现氮输入后期, 氮输入量与土壤矿化水平呈现一定的负相关关系, 即在长期的氮添加试验中, 森林土壤的矿化速率先
随着氮输入量的增加而增大, 继而达到一个峰值, 当氮输入量达到一定水平, 土壤矿化速率便与之呈 现一定的负相关关系。而在本试验中未出现此结果, 可能的原因是草地生态系统与森林生态系统土壤对 养分添加的响应机制不同, 两种生态系统内部结构 差异明显, 植被类型也大不相同, 故而产生不同的 响应; 又因本试验为短期试验, 且是一次性均匀施 肥, 所以结论可能会与Aber和Magill (2004)的研究 有所不同。土壤净硝化速率、净氨化速率和净矿化 速率在处理前均为负值(图3-图5), 其原因可能是土 壤中原有的氮含量较少, 微生物仅可用于维持自身 生长繁殖, 分解更多有机氮对其来说有较大难度, 故不能为土壤无机氮库做出贡献, 因而土壤净硝化 速率、净氨化速率和净矿化速率在处理前均呈现负 值。随着无机氮处理浓度增加, 微生物利用量逐渐 饱和, 故土壤净硝化速率、净氨化速率和净矿化速 率转向正值(白洁冰等, 2011)。

硅虽然是地壳中仅次于氧的第二大元素, 但土 壤中的大部分硅通常会结合其他物质, 形成氧化性 硅酸, 植物无法直接吸收利用, 但硅在植物的生长 过程中发挥着不可或缺的作用, 不仅可以活化土壤 有机质, 促进植物根对氮磷等矿质元素的吸收, 增 加植物抗性(宾振钧等, 2015; 张文鹏等, 2016), 提 高植物叶片的光合作用和植物群落的物种多样性 (Xu et al., 2015, 2018), 还可以缓解环境中污染物, 如可抑制植物光合作用的镉对植物的损害, 且硅对 植物的解毒作用因物种、品种及生态型的不同而表 现出不同的解毒机制(刘彩风等, 2017)。本研究结果 表明, 氮硅配施使土壤中的有效氮含量显著增加, 且显著高于单独施氮时的含量, 土壤矿化活动也较 单独施氮时活跃, 即土壤净硝化速率、净氨化速率 
和净矿化速率均高于单独施氮时。说明施加硅肥能 促进土壤矿化行为, 硅元素对高等植物的矿质营养 有重要影响, 该结果与Fu等(2001)对水稻的研究结 果一致。

与单独氮肥添加相比, 氮硅配施不但能提高不 同土层的氮含量, 而且能促进土壤氮的矿化作用, 但在本研究中, 只涉及一种浓度的硅, 即 $\mathrm{Si} 4$ 与不同 浓度氮肥配施, 进而对土壤矿化作用产生影响。若 要进一步研究硅肥与氮肥对土壤矿化的交互作用, 需在本试验基础上, 增设多个硅浓度。在后续的研 究中可进一步提出在多种氮硅组合中, 是否存在一 个最佳氮硅组合, 使得土壤矿化作用达到最优, 继 而将其作为衡量草地生态系统的养分循环是否良好 及生态系统平衡与否的有效指标。但目前的研究多 集中于硅添加对植被地上生物量、生物多样性、群 落结构等方面的影响, 对硅肥作用的研究也多集中 于其对植物在各种胁迫条件下的抗性、缓解金属污 染物对植物损害等方面, 少有研究涉及其对土壤矿 化作用的影响, 尤其是和氮肥配施对土壤矿化的促 进作用。因此, 养分添加对青藏高原地区土壤氮矿 化的影响还有待更广泛、更深入的研究。

致谢 感谢兰州大学生命科学学院理论生态所的所 有老师、同学在试验中给予的指导与帮助。

\section{参考文献}

Aber JD, Magill AH (2004). Chronic nitrogen additions at the Harvard Forest (USA): The first 15 years of a nitrogen saturation experiment. Forest Ecology and Management, 196, 1-5.

Aber JD, Melillo JM (2001). Terrestrial Ecosystem. 2nd edn. Harcourt Academic Press, San Diego, USA.

Aber JD, Nadelhoffer KJ, Steudler P, Melillo JM (1989). Nitrogen saturation in northern forest ecosystems hypotheses and implications. Bioscience, 39, 378-386.

Bai JB, Xu XL, Fu G, Song MH, He YT, Jiang J (2011). Effects of temperature and nitrogen input on nitrogen mineralization in alpine soils on the Tibetan Plateau. Journal of Anhui Agriculture Science, 39, 14698-14700, 14756. [白 洁冰, 徐兴良, 付刚, 宋明华, 何永涛, 蒋婧 (2011). 温 度和氮素输入对青藏高原3 种高寒草地土壤氮矿化的影 响. 安徽农业科学, 39, 14698-14700, 14756.]

Bai JB, Xu XL, Song MH, He YT, Jiang J, Shi PL (2011). Effects of temperature and added nitrogen on carbon mineralization in alpine soils on the Tibetan Plateau. Ecology and Environmental Sciences, 20, 855-859. [白洁 冰, 徐兴良, 宋明华, 何永涛, 蒋婧, 石培礼 (2011). 温
度和氮素输入对青藏高原三种高寒草地土壤碳矿化的 影响. 生态环境学报, 20,855-859.]

Bin ZJ, Zhang RY, Zhang WP, Xu DH (2015). Effects of nitrogen, phosphorus and silicon addition on leaf carbon, nitrogen, and phosphorus concentration of Elymus nutans of alpine meadow on Qinghai-Tibetan Plateau, China. Acta Ecologica Sinica, 35, 4699-4706. [宾振钧, 张仁懿, 张文 鹏, 徐当会 (2015). 氮磷硅添加对青藏高原高寒草甸垂 穗披碱草叶片碳氮磷的影响. 生态学报, 35, 4699-4706.]

Fu JR, Ho A, Kenichi K (2001). Nitrogen mineralization in two paddy soils as affected by silica source addition. Acta Agriculturae Zhejiangensis, 13(3), 133-140.

Gundersen P, Emmett BA, Kjonaas OJ, Koopmans CJ, Tietema A (1998). Impact of nitrogen deposition on nitrogen cycling in forest: A synthesis of NITREX data. Forest Ecology and Management, 101, 37-55.

Guo YJ (2015). Effects of nitrogen Addition on Alpine Meadow Vegetation and soil Nitrogen Supply Capacity in the Qinghai-Xizang Plateau. Master degree dissertation, Lanzhou University, Lanzhou. [郭雅婧 (2015). 氮素添加对 青藏高原高寒草甸植被和土壤氮素供应能力的影响. 硕士学位论文, 兰州大学, 兰州.]

Liu CF, Shi GR, Yu RG, Zhang Z (2017). Eco-physiological mechanisms of silicon-induced alleviation of cadmium toxicity in plants: A review. Acta Ecologica Sinica, 37, 7799-7810. [刘彩风，史刚荣，余如刚，张铮(2017). 硅 缓解植物镉毒害的生理生态机制. 生态学报, 37 , 7799-7810.]

Liu HX, Guo ZG, Guo XH, Zhou XR, Hui WS, Wang KY (2009). Effect of addition of silicon on water use efficiency and yield components of alfalfa under the different soil moisture. Acta Ecologica Sinica, 29, 3075-3080. [刘] 慧霞, 郭正刚, 郭兴华, 周雪荣, 惠文森, 王康英 (2009). 不同土壤水分条件下硅对紫花苜宿水分利用效 率及产量构成要素的影响. 生态学报, 29, 3075-3080.]

Liu XD, Yin GL, Wu J, Chen JG, Ma LX, Shi SL (2015). Effects of nitrogen addition on the physical properties of soil in an alpine meadow on the eastern Qinghai-Tibetan Plateau. Acta Prataculturae Sinica, 24(10), 12-21. [刘晓 东, 尹国丽, 武均, 陈建纲, 马隆喜, 师尚礼 (2015). 青 藏高原东部高寒草甸草地土壤物理性状对氮元素添加 的响应. 草业学报, 24(10), 12-21.]

Liu XY, Long RJ, Shang ZH (2012). Interactive mechanism of service function of alpine rangeland ecosystems in Qinghai-Tibetan Plateau. Acta Ecologica Sinica, 32, 7688-7697. [刘兴元, 龙瑞军, 尚占环 (2012). 青藏高原 高寒草地生态系统服务功能的互作机制. 生态学报, 32, 7688-7697.]

Si XL, Wang WY, Gao XG, Xu DH (2016). Effects of nitrogen and silicon application on leaf nitrogen content and net photosynthetic rate of Elymus nutans in alpine meadow. 
Chinese Journal of Plant Ecology, 40, 1238-1244. [司晓 林, 王文银, 高小刚, 徐当会 (2016). 氮硅添加对高寒 草甸垂穗披碱草叶片全氮含量及净光合速率的影响. 植物生态学报, 40, 1238-1244.]

Xi ZB (2011). Ammonium nitrogen and nitrate nitrogen. Phosphate \& Compound Fertilizer, 26(1), 62-65. [奚振邦 (2011). 硝态氮与铵态氮. 磷肥与复肥, 26(1), 62-65.]

Xu DH, Fang XW, Zhang RY, Gao TP, Bu HY, Du GZ (2015). Influences of nitrogen, phosphorus and silicon addition on plant productivity and species richness in an alpine meadow. AoB Plants, 7, plv125. DOI: 10.1093/aobpla/plv125.

Xu DH, Gao XG, Gao TP, Mou J, Li JH, Bu HY, Zhang RY, Li QX (2018). Interactive effects of nitrogen and silicon addition on growth of five common plant species and structure of plant community in alpine meadow. Catena, $169,80-89$.

Yang XX, Ren F, Zhou HK, He JS (2014). Responses of plant community biomass to nitrogen and phosphorus additions in an alpine meadow on the Qinghai-Xizang Plateau. Chinese Journal of Plant Ecology, 38, 159-166. [杨晓霞, 任飞, 周华坤, 贺金生 (2014), 青藏高原高寒草甸植物 群落生物量对氮、磷添加的响应. 植物生态学报, 38 , 159-166.]

Zhang JQ, Li Q, Ren ZW, Yang X, Wang G (2010). Effects of nitrogen addition on species richness and relationship between species richness and aboveground productivity of alpine meadow of the Qinghai-Tibetan Platen, China.
Chinese Journal of Plant Ecology, 34, 1125-1131. [张杰 琦, 李奇, 任正炜, 杨雪, 王刚. 氮素添加对青藏高原 高寒草甸植物群落物种丰富度及其与地上生产力关系 的影响. 植物生态学报, 34, 1125-1131.]

Zhang WP, Si XL, Wang WY, Gao TP, Xu DH (2016). Effects of short-term nitrogen and silicon addition on aboveground biomass and biodiversity of alpine meadow of the Qinghai-Tibetan Plateau, China. Pratacultural Science, 33, 38-45. [张文鹏, 司晓林, 王文银, 高天鹏, 徐当会 (2016). 氮硅添加对高寒草甸生物量和多样性的影响 一以青藏高原为例. 草业科学, 33, 38-45.]

Zhang YL, Qi W, Zhou CP, Ding MJ, Liu LS, Gao JG, Bai WQ, Wang ZF, Zheng D (2013). Spatial and temporal variability in the net primary production (NPP) of alpine grassland on Tibetan Plateau from 1982 to 2009. Acta Geographica Sinica, 68, 1197-1211. [张镱锂, 祁威, 周 才平, 丁明军, 刘林山, 高俊刚, 摆万奇, 王兆锋, 郑度 (2013). 青藏高原高寒草地净初级生产力(NPP)时空分 异. 地理学报, 68, 1197-1211.]

Zhao Y, Zhang C, Zhao HF, Xu XN (2013). Effects of N and P addition on soil nitrogen mineralization in a subtropical evergreen broadleaved forest. Chinese Journal of Ecology, 32, 1690-1697. [赵阳, 张驰, 赵泣绯, 徐小牛 (2013). 氮磷添加对亚热带常绿阔叶林土壤氮素矿化的影响. 生态学杂志, 32, 1690-1697.]

责任编委: 程晓莉 责任编辑: 李 敏 